\title{
Characterization of Blood-isolated, Penicillin-Nonsusceptible Streptococcus pneumoniae From Children Between 2014 and 2018 in Bojnurd, Iran
}

\author{
Amir Azimian ${ }^{1,}{ }^{*}$, Mahsa Khosrojerdi ${ }^{2}$, Abdollah Kebriaei ${ }^{1}$, Hasan Namdarahmadabad ${ }^{1}$ and Reza \\ Besharati $^{1}$ \\ ${ }^{1}$ Department of Pathobiology and Laboratory Sciences, Faculty of Medicine, North Khorasan University of Medical Sciences, Bojnurd, Iran \\ ${ }^{2}$ Department of Pediatrics, Faculty of Medicine, North Khorasan University of Medical Sciences, Bojnurd, Iran \\ "Corresponding author: Department of Pathobiology and Laboratory Sciences, Faculty of Medicine, North Khorasan University of Medical Sciences, Bojnurd, Iran. Email: \\ amir_azimian2003@yahoo.com
}

Received 2020 November 11; Revised 2021 February 16; Accepted 2021 February 19.

\begin{abstract}
Background: Streptococcus pneumoniae is one of the common bacterial pathogens in pediatrics. In this study, we performed antimicrobial susceptibility testing, serotyping, and molecular typing of blood-isolated strains of pneumococci in Bojnurd.

Objectives: In the current study, blood-isolated, penicillin-nonsusceptible S. pneumoniae strains were subjected to antimicrobial susceptibility testing and typing of capsular polysaccharides using the quelling reaction and PCR method, as well as genotyping using the Multi Locus Sequence Typing (MLST) method.

Methods: In this study, 51 S. pneumoniae strains were isolated from blood samples of children less than five-years-old in 2014 - 2018. Antibiogram was performed using the Kirby-Bauer method. All of the isolates were serotyped by the Quelling reaction and PCR. The MLST method was applied to determine the molecular types.

Results: Our study revealed that the most common serotypes of blood-isolated pneumococci were 19A, 6A/B, 1, 23F, 19F, 14, 15B/C, and 15A, and the common serotypes in Penicillin-nonsusceptible pneumococci (PNSP) isolates were 19F, 19A, 23F, 14, and finally 15A, 6A/B, 1 , and 15B/C. The MLST analysis of PNSP isolates revealed that three highly resistant isolates with MIC $\geq 16$ belonged to Sweden15A25-19A (ST63), Taiwan19F-14-1(ST236), and Taiwan19F-14 (ST236) clones.

Conclusions: Regarding the common serotypes in this study, it seems that PCV-13 is a suitable choice for vaccination in this area. We also observed a high prevalence of PNSP and multi-drug resistant (MDR) strains between 2014 and 2018. It seems that the Taiwan19F14 clone and its related STs played an essential role in the diffusion of antibiotic-resistant S. pneumoniae isolates in Bojnurd.
\end{abstract}

Keywords: Streptococcus pneumoniae, Serogroup, Genotype, Anti-bacterial Agents, Drug Resistance, Vaccines

\section{Background}

Streptococcus pneumoniae, known as pneumococci, can lead to various diseases ranging from sinusitis and acute otitis media to life-threatening diseases, including meningitis and bacteremia (1-3). Moreover, this bacterium circulates among human societies by colonizing the upper respiratory tract of healthy carriers, representing as its major reservoir (4). Infants and under five-year-old children are more susceptible to pneumococcal diseases due to the lack of a mature immune system and frequent colonization by pneumococci (5). Despite the availability of various vaccines and antibiotics, the World Health Organization (WHO) reported that invasive pneumococcal diseases were responsible for approximately 1.6 million deaths world- wide in 2008 (6).

The polysaccharide capsule, responsible for phagocytosis resistance in the absence of type-specific host antibodies, plays a major role in the invasion of S. pneumoniae in the systemic blood circulation (7). Based on the capsular composition, more than 90 serotypes of pneumococci have been recognized, among which a moderate number of serotypes are related to major infections (8). For the prevention of pneumococcal diseases, serotype-specific pneumococcal capsular polysaccharide vaccines and pneumococcal conjugate vaccines have been designed. These vaccines are composed of the capsular polysaccharides of the most prevalent virulent serotypes. Two types of vaccines are available in the market, including PCV-10 and PCV-13. 
The PCV-10 type covers various serotypes $(1,4,5,6 \mathrm{~B}, 7 \mathrm{~F}, 9 \mathrm{~V}$, $14,18 \mathrm{C}, 19 \mathrm{~F}$, and $23 \mathrm{~F}$ ) while immunity to 13 serotypes is provided by PCV-13 (1, 3, 4, 5, 6A, 6B, 7F, 9V, 14, 18C, 19A, 19F, and 23F(9).

The WHO recommends general immunization against this pathogen as the best preventive strategy against pneumococcal diseases (10). In Iran, a national vaccination program is not currently initiated, and people who want to be vaccinated for pneumococci must do it at their cost. Thus, we need widespread monitoring of serotype diversity of $S$. pneumoniae in Iran to determine the requirement of these vaccines. In recent decades, the treatment of pneumococcal diseases has been complicated due to the increasing penicillin-resistant S. pneumoniae strains (11). The first line of treatment for antibiotic therapy of pneumococcal infections is beta-lactam antibiotics. The first case of penicillin non-susceptible pneumococci (PNSP) was reported in 1967, and since then, resistant strains have been continuously increasing worldwide (12-14). Furthermore, the emergence of multi-drug resistant (MDR) S. pneumoniae isolates has made it more difficult to treat pneumococcal infections. The S. pneumoniae isolates resistant to three or more antimicrobial agents are defined as $\operatorname{MDR}(1,15)$.

\section{Objectives}

Long-term local monitoring of antibiotic resistance in S. pneumoniae can be beneficial for the prevention and control of pneumococcal infections with the correct choice of antibiotics. Therefore, this study focused on the fouryear surveillance of serotypes, antimicrobial resistance, and molecular epidemiology of S. pneumoniae strains isolated from blood cultures of children under five-years-old in Bojnurd, Northeastern Iran, between 2014 and 2018.

\section{Methods}

\subsection{Sample Collection}

This study was conducted from 2014 to 2018 in a major teaching referral Hospital of Bojnurd. All S. pneumoniae strains isolated from blood cultures of non-vaccinated under five-year-old children with non-focal fever suspected endocarditis and acute ill-health were included in this study. A total of 51 isolates were collected. Previously vaccinated children were not entered into this study. It should be noted that in this project, we did not perform additional sampling on patients and used laboratory samples after analysis.

\subsection{Strain Identification}

All blood samples were inoculated into blood culture vials and transported to the Medical Laboratory of Imam Reza Hospital. After 48 -h incubation at $37^{\circ} \mathrm{C}$, the positive cultures were subsequently inoculated onto sheep blood agar plates. Plates were incubated at $37^{\circ} \mathrm{C}$ in $5 \%-10 \% \mathrm{CO}_{2}$ overnight. Suspected colonies were identified based on alpha hemolysis, morphology, gram staining, optochin susceptibility (Oxoid, Basingstoke, UK), and bile solubility tests.

\subsection{Antimicrobial Susceptibility Test}

Susceptibility testing was performed against tetracycline (TET), oxacillin (OXA), trimethoprimsulfamethoxazole (SXT), clindamycin (CLI), erythromycin (ERY), chloramphenicol (CHL), and vancomycin (VAN) for all isolates using the Kirby-Bauer disk diffusion method as per recommendations of the 2017 Clinical and Laboratory Standards Institute (CLSI) (16) using ROSCO antibiotic disks. The strains with an inhibition zone above $20 \mathrm{~mm}$ around the $1-\mu \mathrm{g}$ oxacillin disk test were evaluated as penicillin-resistant. The MIC was determined for these isolates using cefotaxime E-test strips (Biomeriux, France). The interpretive breakpoint used to define penicillin nonsusceptible (PNSP) isolates was $\geq 8$ (penicillin parenteral, non-meningitis) using the E-test method (Biomeriux, France) (16). Besides, S. pneumoniae ATCC 49619 was used as the control strain. A MIC $\geq 8 \mu \mathrm{g} / \mathrm{mL}$ was considered resistant $(11,17,18)$.

\subsection{DNA Extraction}

The total DNA of S. pneumoniae isolates was extracted using a Genet bio blood DNA extraction kit (Genet Bio, Korea). According to kit recommendations, lysozyme (Sigma, Germany) was added at a concentration of $20 \mathrm{IU} / \mathrm{mL}$ to the lysis buffer.

\subsection{Polymerase Chain Reaction}

We confirmed S. pneumoniae isolates using the polymerase chain reaction (PCR). The $l y t A$ and $p s a A$ genes were selected as marker genes to detect S. pneumoniae (19). The PCR was performed using a TAKARA gradient PCR TP600 thermal cycler (TAKARA, Japan). We used Emerald 2X premix Master Mix (TAKARA, Japan) for PCR. The PCR was performed using the following program: $5 \mathrm{~min}$ at $94^{\circ} \mathrm{C}$ for primary denaturation, followed by 35 cycles of amplification (denaturation at $94^{\circ} \mathrm{C}$ for $30 \mathrm{~s}$; annealing at $58^{\circ} \mathrm{C}$ for $30 \mathrm{~s}$; extension at $72^{\circ} \mathrm{C}$ for $30 \mathrm{~s}$ ), and a final extension at $72^{\circ} \mathrm{C}$ for $7 \mathrm{~min}$. The PCR products were evaluated using $1.5 \%$ agarose 
gel electrophoresis. Confirmed isolates were stored at $80^{\circ} \mathrm{C}$ in $15 \%$ sterilized glycerol containing Brain Heart Infusion Broth Media for further analyses (20).

\subsection{Serotyping}

Serotyping was performed using a Quelling reaction with Pneumotest-Latex according to the manufacturer's instructions (Statens Serum Institute, Copenhagen, Denmark). For serotypes $6,15,19$, and 23 , we also performed additional PCR for specific serotypes (21). We set four multiplex PCRs for these serotypes based on the modified Marimon method (22). The PCR thermal cycling condition was $15 \mathrm{~min}$ at $95^{\circ} \mathrm{C}$, followed by 35 cycles of $60 \mathrm{~s}$ at $95^{\circ} \mathrm{C}, 60 \mathrm{~s}$ at $57^{\circ} \mathrm{C}$, and $60 \mathrm{~s}$ at $72^{\circ} \mathrm{C}$, and a final extension step of $10 \mathrm{~min}$ at $72^{\circ} \mathrm{C}$. The PCR products were evaluated using $1.5 \%$ agarose gel electrophoresis.

\subsection{Multi Locus Sequence Typing}

Multilocus sequence typing (MLST) was done using seven housekeeping gene targets, including aroE, gdh, gki, recP, spi, $x p t$, and $d d l$, as described elsewhere (1). The PCR products were subsequently sequenced. The PCR products were sequenced in both directions with an ABI 3730XL DNA analyzer. Sequences were submitted to the MLST database (http://pubmlst.org/spneumonia/), and the STs were determined.

\subsection{Statistical Analysis}

The statistical analysis was performed using Graph Pad Prism 8.0 (version 8.3.0 538). Differences or similarities were evaluated using a two-way ANOVA test. The P-values of $\leq 0.05$ were considered statistically significant.

\section{Results}

\subsection{Antimicrobial Susceptibility}

Most of the S. pneumoniae isolates showed high resistance rates to erythromycin (88.2\%), clindamycin (86.3\%), trimethoprim-sulfamethoxazole (84.3\%), and tetracycline (84.3\%). The resistance rates to chloramphenicol (64.7\%), Oxacillin (35.3\%), and cefotaxime (11.8\%) were relatively low. Notably, all isolates were susceptible to vancomycin. As shown in Table 1, the overall antibiotic resistance trend, except for clindamycin and vancomycin, was increasing from 2014 to 2018. The highest resistance in 2014 - 2015 was to tetracycline, in 2015 - 2016 to clindamycin, in 2016 - 2017 to cefotaxime and oxacillin, and in 2017 - 2018 to cefotaxime. However, the difference in resistance was not statistically significant between the years $(\mathrm{P}=0.057)$.
Antibiotic resistance levels were different in PNSP strains compared to PSSP strains. In the PSSP isolates, resistance against chloramphenicol was 51.6\%, trimethoprimsulfamethoxazole $90.8 \%$, clindamycin $72.6 \%$, erythromycin $93.1 \%$, tetracycline $74.2 \%$, and cefotaxime $6.9 \%$, but in PNSP isolates, resistance to trimethoprim-sulfamethoxazole was $77.8 \%$, clindamycin $100 \%$, erythromycin $83.3 \%$, tetracycline $94.4 \%$, and cefotaxime $16.7 \%$. The difference in resistance was not statistically significant between PNSP and PSSP isolates $(P=0.2227)$. Between different serotypes, the highest resistance to chloramphenicol was observed in 15A, 14, and 19A. All detected serotypes were highly resistant to sulfamethoxazole, clindamycin, erythromycin, and tetracycline. The $19 \mathrm{~F}$ and $15 \mathrm{~A}$ serotypes had low resistance to oxacillin, and all detected serotypes had low resistance to cefotaxime (Table 2). It should be noted that the relationship between serotype and antibiotic resistance was not statistically significant $(P=0.2865)$.

\subsection{Serotype}

Among a total of 51 S. pneumoniae isolates, 50 (98\%) isolates were typable. The most common serotypes were $19 \mathrm{~A}$ (19.6\%), 6A/B (17.64\%), 1(15.68\%), 23F (13.72\%), 19F, 14 and 15B/C (9.8\%), and $15 \mathrm{~A}(1.96 \%)$ but in PNSP isolates, the most common serotypes were $19 \mathrm{~F}, 19 \mathrm{~A}, 23 \mathrm{~F}, 14$, and finally $15 \mathrm{~A}, 6 \mathrm{~A} / \mathrm{B}$, 1 and $15 B / C$, in sequence. Some serotypes such as $19 \mathrm{~F}$ and 15A were just found in PNSP isolates, and some resistances were probably serotype-dependent. The coverage rates of PCV10 and PCV13 were 66.64\% and 86.24\%, respectively.

\subsection{Multi Locus Sequence Typing}

Due to the importance of penicillin non-susceptible pneumococci (PNSP) isolates, we performed Multi Locus Sequence Typing on all of these 18 isolates [f]. The three isolates belonged to international clones Sweden ${ }^{15 \mathrm{~A}}-25-19 \mathrm{~A}$, Taiwan $^{19 \mathrm{~F}}-14-1$, and Taiwan ${ }^{19 \mathrm{~F}}-14$. Two isolates were double locus variant (DLV) of Taiwan ${ }^{19 \mathrm{~F}}-14$ (ST 320), and one isolate was single locus variant SLV (ST271) of this clone. These isolates belonged to serotypes 19A, 1,14, and 6A/B. The four predominant sequence types (ST2687, ST320, ST236, and ST3130) accounted for 50\% of the PNSP isolates (Table 3). Our major complex was clonal complex 0 comprising three STs (236, 271, and 320) with five isolates and clonal complex 1 comprising two STs (63 and 3130) with three isolates. The complete eBURST analysis results are presented in Figures 1 and 2. The differences in antibiotic resistance between various STs were not statistically significant $(\mathrm{P}=$ 0.0636). The distribution of STs was not statistically significant in different serotypes $(\mathrm{P}=0.9990)$. 


\begin{tabular}{|c|c|c|c|c|c|}
\hline \multirow{2}{*}{ Antimicrobial Agents } & \multicolumn{4}{|c|}{ Resistance, No. (\%) } & \multirow{2}{*}{ Total } \\
\hline & $2014-2015$ & $2015-2016$ & $2016-2017$ & 2017-2018 & \\
\hline VAN & $0(0)$ & $0(0)$ & $0(0)$ & $0(0)$ & $0(0)$ \\
\hline TET & $12(27.9)$ & $8(18.6)$ & $10(23.2)$ & $13(30.2)$ & $43(84.3)$ \\
\hline OXA & $1(5.6)$ & $4(22.2)$ & $6(33.3)$ & $7(38.9)$ & $18(35.3)$ \\
\hline ERY & $10(22.2)$ & $11(24.4)$ & $10(22.2)$ & $14(31.1)$ & $45(88.2)$ \\
\hline CLI & $8(18.2)$ & $14(31.8)$ & $9(20.4)$ & $13(29.5)$ & $44(86.3)$ \\
\hline SXT & $4(9.3)$ & $11(25.5)$ & $14(32.6)$ & $14(32.6)$ & $43(84.3)$ \\
\hline CHL & $3(9.1)$ & $9(27.3)$ & $9(27.3)$ & $12(36.3)$ & $33(64.7)$ \\
\hline
\end{tabular}

Abbreviations: CHL, chloramphenicol; CLI, clindamycin; CTX, cefotaxime; ERY, erythromycin; OXA, oxacillin; SXT, trimethoprim-sulfamethoxazole; TET, tetracycline; VAN, vancomycin.

\begin{tabular}{|c|c|c|c|c|c|c|c|c|c|}
\hline & $23 \mathrm{~F}$ & $19 \mathrm{~A}$ & $19 \mathrm{~F}$ & 1 & $6 \mathrm{~A} / \mathrm{B}$ & 14 & $15 \mathrm{~A}$ & $15 \mathrm{~B} / \mathrm{C}$ & NT \\
\hline CHL, \% & 57.1 & 80 & 60 & 0 & 55.6 & 80 & 100 & 60 & 100 \\
\hline SXT,\% & 85.7 & 90 & 100 & 0 & 77.8 & 80 & 0 & 80 & 100 \\
\hline CLI,\% & 85.7 & 90 & 80 & 0 & 77.8 & 80 & 100 & 80 & 100 \\
\hline ERY,\% & 85.7 & 100 & 60 & 87.5 & 88.9 & 100 & 100 & 80 & 100 \\
\hline OXA, \% & 42.9 & 30 & 100 & 12.5 & 11.1 & 40 & 100 & 20 & 100 \\
\hline CTX,\% & 14.3 & 20 & 20 & 25 & 0 & 0 & 0 & 0 & 0 \\
\hline TET,\% & 71.4 & 90 & 80 & 100 & 77.8 & 80 & 100 & 80 & 100 \\
\hline
\end{tabular}

Abbreviations: CLI, clindamycin; CHL, chloramphenicol; CTX, cefotaxime; ERY, erythromycin; OXA, oxacillin; SXT, trimethoprim-sulfamethoxazole; TET, tetracycline.

a All isolates were susceptible to vancomycin

\section{Discussion}

Streptococcus pneumoniae is one of the leading causes of pediatric and adult infections. The serotype distribution, antimicrobial resistance, and genotypes vary across different populations and change over time in each geographical region. Although the results of such studies seem to be of local use, they not only can improve global health by affecting pneumococcal vaccine immunization programs but also raise global awareness about antimicrobial resistance and molecular aspects of pneumococcal infections. This four-year surveillance study revealed that the most common serotypes of blood-isolated pneumococci in our region were 19A, 1, 23F, 19F, 14, and serogroups 15 (B/C) and $6(\mathrm{~A} / \mathrm{B})$, which look like other countries before pneumococcal conjugate vaccination $(15,23,24)$. The PCV10 and PCV13 types covered $66.64 \%$ and $86.24 \%$ of serotypes, respectively, which, like other studies, illustrates that PCV13 could be suitable for controlling S. pneumoniae isolates in this region (25).

All of our isolates were highly resistant to tetracycline, erythromycin, clindamycin, trimethoprimsulfamethoxazole, and chloramphenicol. Contrary, most isolates were susceptible to cefotaxime and penicillin. Of importance, all isolates were susceptible to vancomycin. The highest antimicrobial resistance rate was seen for the 1 and 19A serotypes, while the 6A/B serotype showed the lowest resistance. Table 1 shows that, except for vancomycin, the antimicrobial resistance rate followed an increasing pattern from 2014 to 2018. Penicillin-resistant S. pneumoniae emerged as a global problem in the past decade, and it seems that beta-lactam resistance was increasing during the last few years. In the present study, 35.3\% of the isolates were PNSP, while the overall prevalence of PNSP was lower in other studies. Its prevalence in Morocco was $22.2 \%$ from 2007 to 2014 (26). In other studies, the resistance rate to penicillin in S. pneumoniae was $28 \%$ and $20 \%$ in Tehran, Iran, and in non-meningitis isolates of pneumococci was $10.7 \%$ in Shanghai, China $(1,2,25)$.

Some studies proved that PCV vaccination led to a decrease in the prevalence of antibiotic-resistant strains. Diawara et al. (26) showed that the resistance rate to penicillin decreased from $34.5 \%$ to $22.9 \%$ after PCV vaccination in children. The reduction in the PNSP rate after PCV vaccination was reported in several countries (23, 27-29). Re- 


\begin{tabular}{|c|c|c|c|c|c|c|c|c|c|c|c|c|c|}
\hline \multirow{2}{*}{ Numbers } & \multirow{2}{*}{ Serotype } & \multicolumn{7}{|c|}{ Antimicrobial susceptibility ${ }^{\mathbf{a}}$} & \multirow{2}{*}{ MDR } & \multirow{2}{*}{ MIC, $\mu \mathrm{g} / \mathbf{m L}^{\mathbf{b}}$} & \multirow{2}{*}{ Sequence Type } & \multirow{2}{*}{$\begin{array}{c}\text { Clonal } \\
\text { Complex }\end{array}$} & \multirow{2}{*}{$\begin{array}{l}\text { International } \\
\text { clone }\end{array}$} \\
\hline & & TET & CTX & oxA & ERY & CLI & SXT & CHL & & & & & \\
\hline 1 & $23 \mathrm{~F}$ & $\mathrm{R}$ & s & $\mathrm{R}$ & $\mathrm{R}$ & $\mathrm{R}$ & $\mathrm{R}$ & $\mathrm{R}$ & yes & 8 & 2687 & 7 & - \\
\hline 2 & $19 \mathrm{~A}$ & $\mathrm{R}$ & s & $\mathrm{R}$ & $\mathrm{R}$ & $\mathrm{R}$ & $\mathrm{R}$ & $\mathrm{R}$ & yes & 8 & 276 & 3 & . \\
\hline 6 & 14 & $\mathrm{R}$ & s & $\mathrm{R}$ & $\mathrm{R}$ & $\mathrm{R}$ & s & $\mathrm{R}$ & no & 16 & 143 & 0 & - \\
\hline 8 & $19 \mathrm{~A}$ & $\mathrm{R}$ & $\mathrm{R}$ & $\mathrm{R}$ & $\mathrm{R}$ & $\mathrm{R}$ & $\mathrm{R}$ & $\mathrm{R}$ & yes & 8 & 320 & 0 & $\begin{array}{l}\text { DLV of } \\
\text { Taiwan }^{19 F} \\
-14\end{array}$ \\
\hline 9 & $19 \mathrm{~A}$ & $\mathrm{R}$ & $\mathrm{R}$ & $\mathrm{R}$ & $\mathrm{R}$ & $\mathrm{R}$ & $\mathrm{R}$ & $\mathrm{R}$ & yes & 16 & 320 & 0 & $\begin{array}{l}\text { DLV of } \\
\text { Taiwan }^{19 F} F_{-14}\end{array}$ \\
\hline 18 & $15 \mathrm{~A}$ & $\mathrm{R}$ & s & $\mathrm{R}$ & $\mathrm{R}$ & $\mathrm{R}$ & s & $\mathrm{R}$ & yes & 8 & 63 & 0 & Sweden ${ }^{15 A}-25-19 \mathrm{~A}$ \\
\hline 22 & $19 \mathrm{~F}$ & $\mathrm{R}$ & s & $\mathrm{R}$ & s & $\mathrm{R}$ & $\mathrm{R}$ & s & yes & 8 & 3130 & 0 & - \\
\hline 25 & 14 & $\mathrm{R}$ & s & $\mathrm{R}$ & $\mathrm{R}$ & $\mathrm{R}$ & $\mathrm{R}$ & $\mathrm{R}$ & yes & $\geq 32$ & 329 & 832 & . \\
\hline 27 & $6 \mathrm{~A} / \mathrm{B}$ & $\mathrm{R}$ & s & $\mathrm{R}$ & $\mathrm{R}$ & $\mathrm{R}$ & s & s & yes & $\geq 32$ & 7577 & 2029 & - \\
\hline 28 & $19 \mathrm{~F}$ & $\mathrm{R}$ & s & $\mathrm{R}$ & $\mathrm{R}$ & $\mathrm{R}$ & $\mathrm{R}$ & $\mathrm{R}$ & yes & 16 & 236 & 0 & Taiwan $^{19 \mathrm{~F}_{-14-1}}$ \\
\hline 34 & 1 & $\mathrm{R}$ & s & $\mathrm{R}$ & $\mathrm{R}$ & $\mathrm{R}$ & $\mathrm{R}$ & $\mathrm{R}$ & yes & 8 & 10081 & 84 & . \\
\hline 36 & $23 \mathrm{~F}$ & $\mathrm{R}$ & s & $\mathrm{R}$ & $\mathrm{R}$ & $\mathrm{R}$ & $\mathrm{R}$ & R & yes & 16 & 2687 & 0 & - \\
\hline 38 & $19 \mathrm{~F}$ & S & $\mathrm{R}$ & $\mathrm{R}$ & $\mathrm{R}$ & $\mathrm{R}$ & $\mathrm{R}$ & $\mathrm{R}$ & yes & 8 & 271 & 0 & $\begin{array}{l}\text { SLV of } \\
\text { Taiwan }^{19 F}-14\end{array}$ \\
\hline 41 & $19 \mathrm{~F}$ & $\mathrm{R}$ & s & $\mathrm{R}$ & $\mathrm{R}$ & $\mathrm{R}$ & $\mathrm{R}$ & $\mathrm{R}$ & yes & $\geq 32$ & 236 & 0 & Taiwan $^{19 \mathrm{~F}_{-14}}$ \\
\hline 45 & $23 \mathrm{~F}$ & $\mathrm{R}$ & s & $\mathrm{R}$ & $\mathrm{R}$ & $\mathrm{R}$ & $\mathrm{R}$ & $\mathrm{R}$ & yes & 16 & 2687 & 0 & . \\
\hline 47 & $15 \mathrm{~B} / \mathrm{C}$ & $\mathrm{R}$ & s & $\mathrm{R}$ & s & $\mathrm{R}$ & S & S & yes & 16 & 13823 & 100 & - \\
\hline 49 & $19 \mathrm{~F}$ & $\mathrm{R}$ & s & $\mathrm{R}$ & s & $\mathrm{R}$ & $\mathrm{R}$ & s & yes & $\geq 32$ & 3130 & 0 & - \\
\hline 51 & NT & $\mathrm{R}$ & s & $\mathrm{R}$ & $\mathrm{R}$ & $\mathrm{R}$ & $\mathrm{R}$ & $\mathrm{R}$ & yes & 8 & 9777 & 0 & . \\
\hline
\end{tabular}
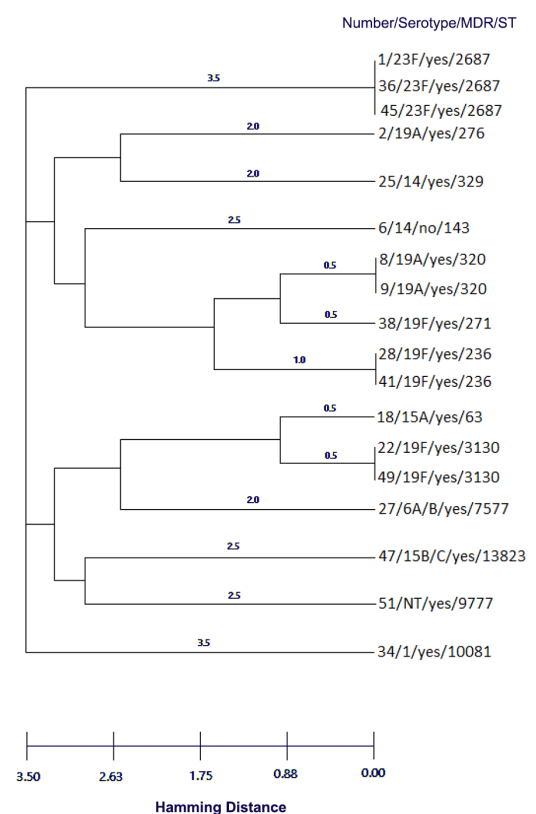

Figure 1. Dendrogram constructed from MLST data showing the genetic relatedness of the 18 pneumococcal sequence types in Table 3. ST320 and ST276 are SLVs of ST271, and also ST63 and ST3130 are SLV. garding relatively high resistance against penicillin in our isolates and also high serotype coverage of the 13-valent vaccine, it seems that the PCV vaccination could be useful to prevent the spread of resistant strains. The most prevalent serotypes among our PNSP were 19F, 19A, 23F, 14, 15B/C, $6 \mathrm{~A} / \mathrm{B}$, and 1. No meaningful correlation was observed between serotypes and penicillin resistance $(\mathrm{P}>0.05)$. These serotypes were mainly covered by PCV-13.

Serotypes 14, 6A/B, and $19 \mathrm{~F}$ were observed among highly resistant isolates with penicillin MICs $\geq 32$. Dissemination of $19 \mathrm{~F}$ isolates has increased in many parts of the world, and they have become resistant to penicillin (3032). In this study, all $19 \mathrm{~F}$ isolates were resistant to penicillin. It should be noted that regardless of antimicrobial resistance, the most prevalent serotypes among our isolates were $19 \mathrm{~A}, 6 \mathrm{~A} / \mathrm{B}, 1,23 \mathrm{~F}, 19 \mathrm{~F}, 14,15 \mathrm{~B} / \mathrm{C}$, and $15 \mathrm{~A}$, respectively. In a similar study in Trinidad and Tobago, the most prevalent serotypes among invasive isolates were $19 \mathrm{~F}, 6 \mathrm{~B}$, $23 \mathrm{~F}, 3,19 \mathrm{~A}, 6 \mathrm{~A}, 14$, and 9V, respectively (33). In another study in China, the most common serotypes were 19F, 19A, 15, 6B, $6 \mathrm{~A}$, and 17 (34). Comparing our results with other studies shows partial differences in various geographical areas (3, 24,35-39); for example, serotype 1 was not reported in China and Trinidad and Tobago studies, but we found it in our iso- 


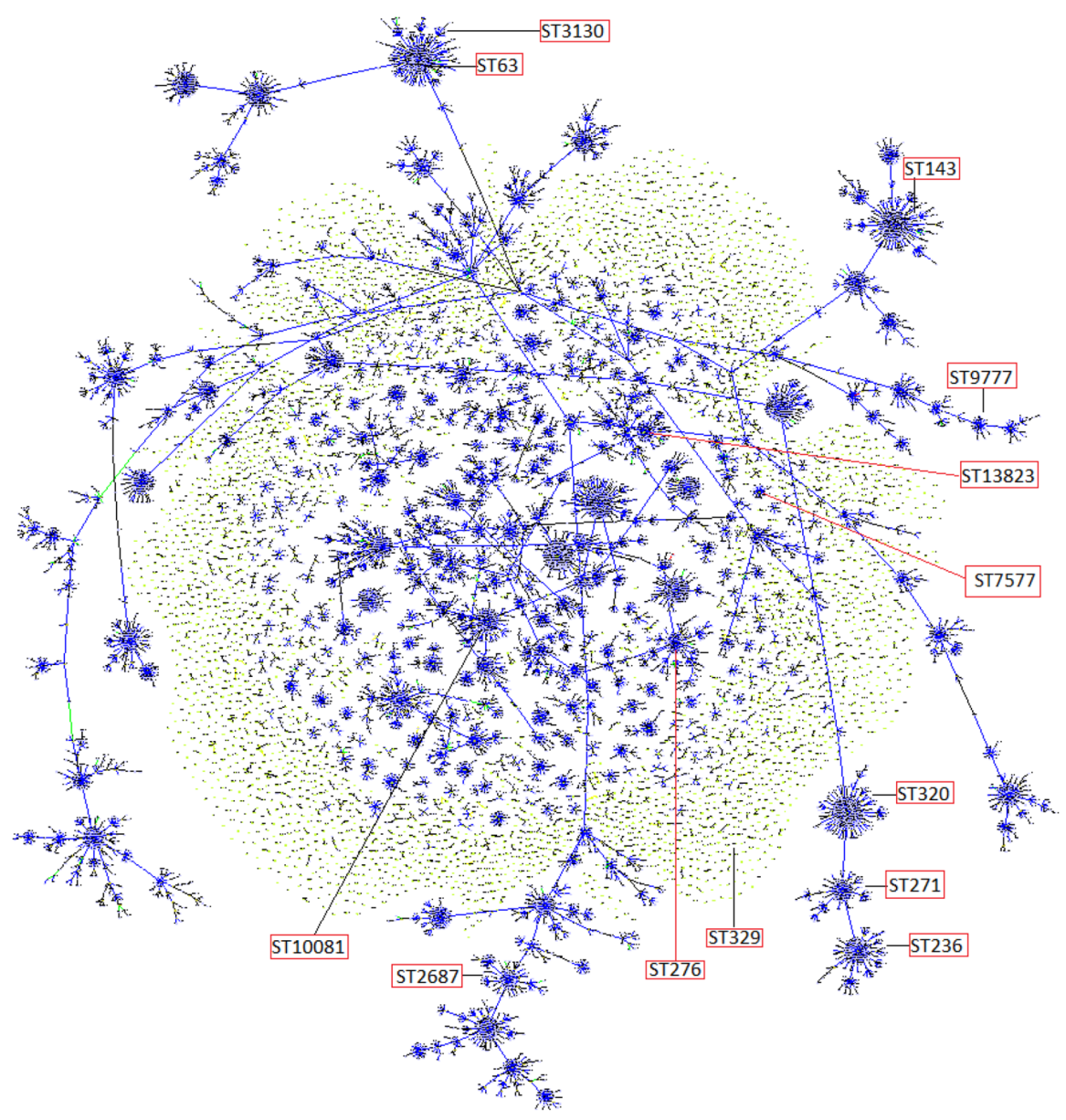

Figure 2. Population snapshot of 12 STs of Streptococcus pneumoniae isolates. Clusters of related STs and individual unlinked STs with the entire S. pneumoniae MLST database (15219 STs) are displayed as a single eBURST diagram by setting the group definition to 0 of 7 shared alleles. STs found in this study are labeled and other ST labels have been removed. Our STs belonged to CC0, CC3, CC84, CC100, CC832, and CC2029.

lates. Conversely, serotypes 3 and 17 were found in that two countries though we did not observe it in our study.

The MLST analysis of S. pneumoniae isolates in different countries showed that some international clones such as Spain $^{23 \mathrm{~F}}-1$, Spain ${ }^{6 \mathrm{~B}}-2$, or England ${ }^{14}-9$ are circulating worldwide $(1,40,41)$. The MLST analysis of PNSP isolates in this study revealed that three highly penicillin-resistant isolates with MIC $\geq 16$ belonged to international clones, Sweden15A-25-19A (ST63), Taiwan19F-14-1, and Taiwan19F-14 (ST236). Importantly, for our Taiwan19F-14-1 and Taiwan19F14 isolates, the MICs were higher than previously reported (41). In primary introduced Taiwan19F-14-1 and Taiwan19F14 , the penicillin MIC was 2, but in our isolates and variants, the MIC was minimally $\geq 8$. Other prevalent STs in our isolates were ST2687, ST320, and ST3130. It should be noted that ST320 is a DLV, and ST271 is an SLV of the ST236 Taiwan19F-14 clone (Figure 1). The spread of ST320 can be problematic because it is an international virulent MDR strain (42). This strain has been reported in many countries and is commonly found in North America, Europe, China, and other Asian countries $(2,15,42,43)$. All of our ST320 isolates belonged to serotype 19A (Figure 1).

In the present study, $82.35 \%$ of the isolates were classified as MDR. The relatively high presence of Taiwan19F-14 clones and their related STs put them as significant clones in circulation. Talebi et al. (2) reported that common STs in erythromycin-resistant isolates of pneumococci were ST3130, ST180, and ST81. According to the results of another study by Raddoui et al. (44), ST81 was the most prevalent sequence type in macrolide-resistant S. pneumoniae isolates. 
Between 2007 and 2013, the most common ST in MDR pneumococci isolates was ST320 in Canada (15). Lucas et al. (33) reported that ST138, ST36, and ST180 were the most common sequence types in Trinidad and Tobago, while in this study, the most common STs in MDR isolates were 2687, $320,236,3130,271,329,63,7577,10081,13823$, and 9777, respectively.

Serotype switching was observed in serotype 19A PNSP belonging to ST320 and ST276, serotype $19 \mathrm{~F}$ distributed in ST3130, ST271, and ST236, and serotype 14 found in ST329 and ST143. A high rate of capsular switching was reported in other studies, especially in regions with national vaccination programs. Interestingly, we observed a high level of serotype switching in our isolates, although, in Iran, the state-run vaccination program has not been carried out yet. Therefore, further studies are required to confirm the relationship between serotype switching and vaccination. Finally, it seems that our PNSP isolates belonged to diverse clones that may be due to the various races living in this geographical area (Figure 2).

\subsection{Conclusions}

In conclusion, the current study showed that serotypes $19 \mathrm{~A}, 6 \mathrm{~A} / \mathrm{B}, 1,23 \mathrm{~F}, 19 \mathrm{~F}, 14$, and $15 \mathrm{~B} / \mathrm{C}$ were commonly isolated from blood cultures of children under five-years-old. It seems that PCV-13 with the $86.24 \%$ coverage of blood isolates of pneumococci is the most suitable choice for vaccination in this region. Other important points are a relatively high prevalence of PNSP and MDR strains between 2014 and 2018. The MLST surveillance showed that the Taiwan ${ }^{19 \mathrm{~F}}-14$ clone and its related STs played an essential role in disseminating resistant $S$. pneumoniae isolates in Bojnurd, Iran. Hence, to select the optimal antimicrobial treatment, to understand the distribution pattern of clones, and to have the most effective immunization program for S. pneumoniae-related diseases, long-term regional surveillance could be beneficial.

\section{Footnotes}

Authors' Contribution: Amir Azimian conceptualized and designed the study, carried out the conventional and PCR tests, wrote the initial draft, and conducted the final revision of the manuscript. Mahsa Khosrojerdi collected data, carried out initial analyses, reviewed, and revised the manuscript. Abdollah Kebriaei collected laboratory data, edited, and revised the manuscript. Hasan NamdarAhmadabad collected laboratory data, edited, and revised the manuscript. Reza Besharati collected laboratory data.
All authors approved the final manuscript as submitted and agreed to be accountable for all aspects of the work.

Conflict of Interests: The authors declare that they have no conflict of interest.

Ethical Approval: We received Ethics Committee approval numbered IR.nkums.REC.1395.107 before beginning this project.

Funding/Support: The grant number was 95/P/970.

\section{References}

1. Pan F, Han L, Huang W, Tang J, Xiao S, Wang C, et al. Serotype distribution, antimicrobial susceptibility, and molecular epidemiology of streptococcus pneumoniae isolated from children in Shanghai, China. PLoS One. 2015;10(11). e0142892. doi: 10.1371/journal.pone.0142892. [PubMed: 26571373]. [PubMed Central: PMC4646667].

2. Talebi M, Azadegan A, Sadeghi J, Ahmadi A, Ghanei M, Katouli M, et al. Determination of characteristics of erythromycin resistant streptococcus pneumoniae with preferred PCV usage in Iran. PLoS One. 2016;11(12). e0167803. doi: 10.1371/journal.pone.0167803. [PubMed: 28033345]. [PubMed Central: PMC5199012].

3. Houri H, Tabatabaei SR, Saee Y, Fallah F, Rahbar M, Karimi A. Distribution of capsular types and drug resistance patterns of invasive pediatric Streptococcus pneumoniae isolates in Teheran, Iran. Int J Infect Dis. 2017;57:21-6. doi: 10.1016/j.ijid.2017.01.020. [PubMed: 28131730].

4. Pinto TC, Kegele FC, Dias CA, Barros RR, Peralta JM, Merquior VL, et al. Streptococcus pneumoniae serotypes 9 and 14 circulating in Brazil over a 23-year period prior to introduction of the 10valent pneumococcal conjugate vaccine: Role of international clones in the evolution of antimicrobial resistance and description of a novel genotype. Antimicrob Agents Chemother. 2016;60(11):6664-72. doi: 10.1128/AAC.00673-16. [PubMed: 27572394]. [PubMed Central: PMC5075071].

5. Centers for Disease Control Prevention. Invasive pneumococcal disease in young children before licensure of 13-valent pneumococcal conjugate vaccine - United States, 2007. MMWR Morb Mortal Wkly Rep. 2010;59(9):253-7. [PubMed: 20224541].

6. Black RE, Cousens S, Johnson HL, Lawn JE, Rudan I, Bassani DG, et al. Global, regional, and national causes of child mortality in 2008: A systematic analysis. Lancet. 2010;375(9730):1969-87. doi: 10.1016/S01406736(10)60549-1. [PubMed: 20466419].

7. Kadioglu A, Weiser JN, Paton JC, Andrew PW. The role of Streptococcus pneumoniae virulence factors in host respiratory colonization and disease. Nat Rev Microbiol. 2008;6(4):288-301. doi: 10.1038/nrmicro1871. [PubMed: 18340341].

8. Hausdorff WP, Bryant J, Paradiso PR, Siber GR. Which pneumococcal serogroups cause the most invasive disease: implications for conjugate vaccine formulation and use, part I. Clin Infect Dis. 2000;30(1):100-21. doi: 10.1086/313608. [PubMed: 10619740].

9. Miller E, Andrews NJ, Waight PA, Slack MP, George RC. Effectiveness of the new serotypes in the 13-valent pneumococcal conjugate vaccine. Vaccine. 2011;29(49):9127-31. doi: 10.1016/j.vaccine.2011.09.112. [PubMed: 21983361].

10. Hansen J, Black S, Shinefield H, Cherian T, Benson J, Fireman B, et al. Effectiveness of heptavalent pneumococcal conjugate vaccine in children younger than 5 years of age for prevention of pneumonia: Updated analysis using World Health Organization standardized inter- 
pretation of chest radiographs. Pediatr Infect Dis J. 2006;25(9):779-81. doi: 10.1097/01.inf.0000232706.35674.2f. [PubMed: 16940833].

11. Ruhe JJ, Myers L, Mushatt D, Hasbun R. High-level penicillinnonsusceptible Streptococcus pneumoniae bacteremia: identification of a low-risk subgroup. Clin Infect Dis. 2004;38(4):508-14. doi: 10.1086/381197. [PubMed: 14765343].

12. Hansman D, Bullen MM. A resistant pneumococcus. Lancet. 1967;290(7509):264-5. doi: 10.1016/s0140-6736(67)92346-x.

13. Jones RN, Sader HS, Mendes RE, Flamm RK. Update on antimicrobial susceptibility trends among Streptococcus pneumoniae in the United States: Report of ceftaroline activity from the SENTRY Antimicrobial Surveillance Program (1998-2011). Diagn Microbiol Infect Dis. 2013;75(1):107-9. doi: 10.1016/j.diagmicrobio.2012.08.024. [PubMed: 23009730].

14. Kim SH, Song JH, Chung DR, Thamlikitkul V, Yang Y, Wang H, et al. Changing trends in antimicrobial resistance and serotypes of Streptococcus pneumoniae isolates in Asian countries: An Asian Network for Surveillance of Resistant Pathogens (ANSORP) study. Antimicrob Agents Chemother. 2012;56(3):1418-26. doi: 10.1128/AAC.05658-11. [PubMed: 22232285]. [PubMed Central: PMC3294909].

15. Golden AR, Rosenthal M, Fultz B, Nichol KA, Adam HJ, Gilmour MW, et al. Characterization of MDR and XDR Streptococcus pneumoniae in Canada, 2007-13. J Antimicrob Chemother. 2015;70(8):2199-202. doi: 10.1093/jac/dkv107. [PubMed: 25921512].

16. Wayne P. Performance standards for Antimicrobial Susceptibility testing; 24th informational supplement. Clinical and Laboratory Standards Institute (CLSI); 2017.

17. Imohl M, Reinert RR, Tulkens PM, van der Linden M. Penicillin susceptibility breakpoints for Streptococcus pneumoniae and their effect on susceptibility categorisation in Germany (1997-2013). Eur J Clin Microbiol Infect Dis. 2014;33(11):2035-40. doi: 10.1007/s10096-014-2174z. [PubMed: 24930041].

18. Weinstein MP, Klugman KP, Jones RN. Rationale for revised penicillin susceptibility breakpoints versus Streptococcus pneumoniae: Coping with antimicrobial susceptibility in an era of resistance. Clin Infect Dis. 2009;48(11):1596-600. doi: 10.1086/598975. [PubMed: 19400744].

19. Jourdain S, Dreze PA, Vandeven J, Verhaegen J, Van Melderen L, Smeesters PR. Sequential multiplex PCR assay for determining capsular serotypes of colonizing S. pneumoniae. BMC Infect Dis. 2011;11:100. doi: 10.1186/1471-2334-11-100. [PubMed: 21507244]. [PubMed Central: PMC3094224].

20. Havaei SA, Halaji M, Vidovic S, R. Dillon J, Karbalaei M, Ghanbari F, et al. Prevalence and genotyping of methicillin-resistant and - susceptible Staphylococcus aureus strains isolated from patients in a University Hospital, Isfahan, Iran. Jundishapur J Microbiol. 2017;10(5). doi: 10.5812/jjm.13571.

21. Abdoli S, Safamanesh S, Khosrojerdi M, Azimian A. Molecular detection and serotyping of Streptococcus pneumoniae in children with suspected meningitis in Northeast Iran. Iran J Med Sci. 2020;45(2):12533. doi: 10.30476/IJMS.2019.45423. [PubMed: 32210489]. [PubMed Central: PMC7071549].

22. Marimon JM, Ercibengoa M, Santacatterina E, Alonso M, Perez-Trallero E. Single-step Multiplex PCR Assay for determining 92 pneumococcal serotypes. J Clin Microbiol. 2016;54(8):2197-200. doi: 10.1128/JCM.0115616. [PubMed: 27280423]. [PubMed Central: PMC4963499].

23. Richter SS, Heilmann KP, Dohrn CL, Riahi F, Diekema DJ, Doern GV. Pneumococcal serotypes before and after introduction of conjugate vaccines, United States, 1999-2011(1.). Emerg Infect Dis. 2013;19(7):107483. doi: 10.3201/eid1907.121830. [PubMed: 23763847]. [PubMed Central: PMC3713983].

24. Tai SS. Streptococcus pneumoniae Serotype distribution and pneumococcal conjugate vaccine serotype coverage among pediatric patients in East and Southeast Asia, 2000-2014: A pooled data analysis.
Vaccines (Basel). 2016;4(1). doi: 10.3390/vaccines4010004. [PubMed: 26907356]. [PubMed Central: PMC4810056].

25. Talebi M, Sadeghi J, Ahmadi A, Lohrasbi V, Owlia P, Pourshafie MR. High rate of serotype switching and genetic variations indicates widespread recombination between clinical and commensal penicillin-nonsusceptible Streptococcus pneumoniae in Tehran. Microb Drug Resist. 2019;25(6):865-73. doi: 10.1089/mdr.2018.0336. [PubMed: 30785836].

26. Diawara I, Barguigua A, Katfy K, Nayme K, Belabbes H, Timinouni M, et al. Molecular characterization of penicillin non-susceptible Streptococcus pneumoniae isolated before and after pneumococcal conjugate vaccine implementation in Casablanca, Morocco. Ann Clin Microbiol Antimicrob. 2017;16(1):23. doi: 10.1186/s12941-017-0200-6. [PubMed: 28376809]. [PubMed Central: PMC5381081].

27. Demczuk WH, Martin I, Griffith A, Lefebvre B, McGeer A, Lovgren $M$, et al. Serotype distribution of invasive Streptococcus pneumoniae in Canada after the introduction of the 13-valent pneumococcal conjugate vaccine, 2010-2012. Can J Microbiol. 2013;59(12):778-88. doi: 10.1139/cjm-2013-0614. [PubMed: 24313450].

28. von Gottberg A, de Gouveia L, Tempia S, Quan V, Meiring S, von Mollendorf C, et al. Effects of vaccination on invasive pneumococcal disease in South Africa. N Engl J Med. 2014;371(20):1889-99. doi: 10.1056/NEJMoa1401914. [PubMed: 25386897].

29. dos Santos SR, Passadore LF, Takagi EH, Fujii CM, Yoshioka CR, Gilio $\mathrm{AE}$, et al. Serotype distribution of Streptococcus pneumoniae isolated from patients with invasive pneumococcal disease in Brazil before and after ten-pneumococcal conjugate vaccine implementation. Vaccine. 2013;31(51):6150-4. doi: 10.1016/j.vaccine.2013.05.042. [PubMed: 23747454].

30. Gounder PP, Bruden D, Rudolph K, Zulz T, Hurlburt D, Thompson G, et al. Re-emergence of pneumococcal colonization by vaccine serotype $19 \mathrm{~F}$ in persons aged $>\mid=5$ years after 13-valent pneumococcal conjugate vaccine introduction-Alaska, 2008-2013. Vaccine. 2018;36(5):6917. doi: 10.1016/j.vaccine.2017.12.035. [PubMed: 29279284].

31. Oodate M, Kimura K, Banno H, Yokoyama S, Jin W, Wachino JI, et al. Predominance of serogroup 19 CC320/271 among penicillinnonsusceptible Streptococcus pneumoniae isolates after introduction of the PCV7 vaccine in several regions of Japan. Jpn J Infect Dis. 2018;71(1):14-20. doi: 10.7883/yoken.JJID.2017.236. [PubMed: 29093321].

32. Zemlickova H, Malisova L, Spanelova P, Jakubu V, Kozakova J, Musilek $\mathrm{M}$, et al. Molecular characterization of serogroup 19 Streptococcus pneumoniae in the Czech Republic in the post-vaccine era. J Med Microbiol. 2018;67(7):1003-11. doi: 10.1099/jmm.0.000765. [PubMed: 29856703]. [PubMed Central: PMC6152367].

33. Nurse-Lucas M, McGee L, Hawkins PA, Swanston WH, Akpaka PE. Serotypes and genotypes of Streptococcus pneumoniae isolates from Trinidad and Tobago. Int J Infect Dis. 2016;46:100-6. doi: 10.1016/j.ijid.2016.04.005. [PubMed: 27062986]. [PubMed Central: PMC5739879].

34. Zhao C, Li Z, Zhang F, Zhang X, Ji P, Zeng J, et al. Serotype distribution and antibiotic resistance of Streptococcus pneumoniae isolates from 17 Chinese cities from 2011 to 2016. BMC Infect Dis. 2017;17(1):804. doi: 10.1186/s12879-017-2880-0. [PubMed: 29284419]. [PubMed Central: PMC5747162].

35. Johnson HL, Deloria-Knoll M, Levine OS, Stoszek SK, Freimanis Hance L, Reithinger R, et al. Systematic evaluation of serotypes causing invasive pneumococcal disease among children under five: The pneumococcal global serotype project. PLoS Med. 2010;7(10). doi: 10.1371/journal.pmed.1000348. [PubMed: 20957191]. [PubMed Central: PMC2950132].

36. Le CF, Palanisamy NK, Mohd Yusof MY, Sekaran SD. Capsular serotype and antibiotic resistance of Streptococcus pneu- 
moniae isolates in Malaysia. PLoS One. 2011;6(5). e19547. doi: 10.1371/journal.pone.0019547. [PubMed: 21603602]. [PubMed Central: PMC3095606].

37. Gharailoo Z, Mousavi SF, Halvani N, Feizabadi MM. Antimicrobial resistant pattern and capsular typing of streptococcus pneumoniae isolated from children in Sistan -Baluchestan. Maedica (Bucur). 2016;11(3):203-7. [PubMed: 28694854]. [PubMed Central: PMC5486161].

38. Bokaeian M, Khazaei HA, Javadimehr M. Nasopharyngeal carriage, antibiotic resistance and serotype distribution of Streptococcus pneumoniae among healthy adolescents in Zahedan. Iran Red Crescent Med J. 2011;13(5):328-33. [PubMed: 22737489]. [PubMed Central: PMC3371970].

39. Soto-Nogueron A, Carnalla-Barajas MN, Solorzano-Santos F, Arrendondo-Garcia JL, Arzate-Barbosa P, Tinoco-Favila JC, et al. Streptococcus pneumoniae as cause of infection in infants less than 60 days of age: Serotypes and antimicrobial susceptibility. Int J Infect Dis. 2016;42:69-73. doi: 10.1016/j.ijid.2015.12.001. [PubMed: 26673859].

40. Ip M, Lyon DJ, Yung RW, Tsang L, Cheng AF. Introduction of new clones of penicillin-nonsusceptible Streptococcus pneumoniae in Hong Kong. J Clin Microbiol. 2002;40(4):1522-5. doi: 10.1128/jcm.40.4.1522-
1525.2002. [PubMed: 11923387]. [PubMed Central: PMC140348].

41. McGee L, McDougal L, Zhou J, Spratt BG, Tenover FC, George R, et al. Nomenclature of major antimicrobial-resistant clones of Streptococcus pneumoniae defined by the pneumococcal molecular epidemiology network. J Clin Microbiol. 2001;39(7):2565-71. doi: 10.1128/JCM.39.7.2565-2571.2001. [PubMed: 11427569]. [PubMed Central: PMC88185].

42. Ardanuy C, Rolo D, Fenoll A, Tarrago D, Calatayud L, Linares J. Emergence of a multidrug-resistant clone (ST320) among invasive serotype 19A pneumococci in Spain. JAntimicrob Chemother. 2009;64(3):507-10. doi: 10.1093/jac/dkp210. [PubMed: 19535383].

43. Shin J, Baek JY, Kim SH, Song JH, Ko KS. Predominance of ST320 among Streptococcus pneumoniae serotype 19A isolates from 10 Asian countries. J Antimicrob Chemother. 2011;66(5):1001-4. doi: 10.1093/jac/dkr048. [PubMed: 21393143].

44. Raddaoui A, Tanfous FB, Chebbi Y, Achour W, Baaboura R, Benhassen A. High prevalence of multidrug-resistant international clones among macrolide-resistant Streptococcus pneumoniae isolates in immunocompromised patients in Tunisia. Int J Antimicrob Agents. 2018;52(6):893-7. doi: 10.1016/j.ijantimicag.2018.04.015. [PubMed: 29698665]. 